\title{
ESTIMATION OF DILATATIONS FOR MAPPINGS MORE GENERAL THAN QUASIREGULAR MAPPINGS
}

\author{
R. R. Salimov and E.A. Sevost'yanov
}

UDC 517.5

\begin{abstract}
We consider the so-called ring $Q$-mappings, which are natural generalizations of quasiregular mappings in a sense of Väisälä's geometric definition of moduli. It is shown that, under the condition of nondegeneracy of these mappings, their inner dilatation is majorized by a function $Q(x)$ to within a constant depending solely on the dimension of the space.
\end{abstract}

\section{Introduction}

It is known that the geometric definition of quasiconformal mappings in a domain $D$ from $\mathbb{R}^{n}, n \geq 2$, proposed by Väisälä is based on the condition

$$
M(f(\Gamma)) \leq K M(\Gamma)
$$

for any family $\Gamma$ of curves $\gamma$ in the domain $D$, where $M$ is a conformal modulus of the family of curves (outer measure defined on the families of curves in $\mathbb{R}^{n}$ ) and $K \geq 1$ is a constant (see Definition 13.1, Sec. 13 of Chap. II, and Theorem 34.3 in Chap. IV in [1]). In other words, the modulus of any family of curves under quasiconformal mappings is never distorted by more than a factor of $K$, where $K<\infty$ is a constant. The following assertion is proved in [1] (Theorem 34.6, Chap. IV):

Proposition 1. Assume that the homeomorphism $f: D \rightarrow \overline{\mathbb{R}^{n}}, n \geq 2$, satisfies condition (1) in the domain $D$ for any family of curves $\Gamma$. Then the following inequality holds for almost all $x \in D$ :

$$
|J(x, f)| \leq K \cdot l\left(f^{\prime}(x)\right)
$$

where $J(x, f)$ is the Jacobian of the mapping $f$ at a point $x$ and

$$
l\left(f^{\prime}(x)\right):=\min _{h \in \mathbb{R}^{n} \backslash\{0\}} \frac{\left|f^{\prime}(x) h\right|}{|h|} .
$$

Proposition 1 plays an important role in the theory of quasiconformal mappings because it relates the pure geometric condition (1) with an analytic condition of the form (2).

We now assume that the definition of the analyzed class of mappings is based, instead of relation (1), on an inequality of the form

Institute for Applied Mathematics and Mechanics, National Academy of Sciences of Ukraine, Donetsk, Ukraine.

Translated from Ukrains'kyi Matematychnyi Zhurnal, Vol. 62, No. 11, pp. 1531-1537, November, 2010. Original article submitted February $12,2010$. 


$$
M(f(\Gamma)) \leq \int_{D} Q(x) \cdot \rho^{n}(x) d m(x),
$$

where $m$ is the Lebesgue measure in $\mathbb{R}^{n}, \rho$ is an arbitrary nonnegative Borel function such that the length of an arbitrary curve $\gamma$ from the family $\Gamma$ measured in the metric $\rho$ is not smaller than 1 , i.e.,

$$
\int_{\gamma} \rho(x)|d x| \geq 1
$$

for all curves $\gamma \in \Gamma$, and $Q: D \rightarrow[1, \infty]$ is a given Lebesgue measurable function specified in advance (see, e.g., [2]).

We define the inner dilatation $K_{I}(x, f)$ of a mapping $f$ as follows:

$$
K_{I}(x, f)=\frac{|J(x, f)|}{l\left(f^{\prime}(x)\right)^{n}}
$$

if the Jacobian

$$
J(x, f):=\operatorname{det} f^{\prime}(x) \neq 0,
$$

$K_{I}(x, f)=1$ for $f^{\prime}(x)=0$, and $K_{I}(x, f)=\infty$ if the Jacobi matrix $f^{\prime}(x)$ of the mapping $f$ at a point $x$ is nonzero but the Jacobian $J(x, f)=0$.

The main result of the present paper is the following assertion:

Proposition 2. Assume that an open discrete mapping $f: D \rightarrow \overline{\mathbb{R}^{n}}, n \geq 2$, satisfies an estimate of the form (3) for some Lebesgue measurable function $Q \in L_{\mathrm{loc}}^{1}$. Then the inequality

$$
K_{I}(x, f) \leq c_{n} \cdot Q(x)
$$

is true for almost all $x \in D$ and a constant $c_{n}$ depending only on $n$.

\section{Preliminaries}

It seems likely that, for the first time, an inequality of the form (3) was established by Lehto and Virtanen [3, p. 221] (Sec. 6.3, Chap. V) for quasiconformal mappings in the plane and by Strugov [4] for the so-called mappings quasiconformal in the mean in the space. Relation (3) was established by Gutlyanskii (together with Bishop, Martio, and Vuorinen) in [5] for the quasiconformal mappings in the space; in this case, $Q$ was equal to $K_{I}(x, f)$. In [6], Miklyukov studied some other classes of mappings satisfying the estimates characterized by certain similarities with the inequality presented above.

In what follows, we assume that not all curves of the family $\Gamma$ but only some of them are distorted in a controlled way. Indeed, we restrict ourselves to the analysis of the families of curves $\Gamma$ connecting concentric spheres centered at a fixed point of a given domain. For our purposes (in particular, to prove Proposition 2), it suffices to consider only the indicated families $\Gamma$. An inequality of the form (3) satisfied for these families of curves serves as a basis of the definition of ring $Q$-mappings (see, e.g., [2, Sec. 7.1, Chap. VII]).

Throughout the paper, $D$ is a domain in $\mathbb{R}^{n}, n \geq 2$,

$$
B\left(x_{0}, r\right)=\left\{x \in \mathbb{R}^{n}:\left|x-x_{0}\right|<r\right\}, \quad S\left(x_{0}, r\right)=\left\{x \in \mathbb{R}^{n}:\left|x-x_{0}\right|=r\right\},
$$




$$
A\left(r_{1}, r_{2}, x_{0}\right)=\left\{x \in \mathbb{R}^{n}: r_{1}<\left|x-x_{0}\right|<r_{2}\right\}
$$

$m$ is the Lebesgue measure in $\mathbb{R}^{n}$, and $\Omega_{n}$ is the volume of the unit ball $\mathbb{B}^{n}:=B(0,1)$ in $\mathbb{R}^{n}$. The expression $f: D \rightarrow \mathbb{R}^{n}$ means that a mapping $f$ is defined in the domain $D$ and is continuous. The mapping $f: D \rightarrow \mathbb{R}^{n}$ is called discrete if the preimage $f^{-1}(y)$ of every point $y \in \mathbb{R}^{n}$ consists of isolated points. This mapping is called open if the image of any open set $U \subset D$ is an open set in $\mathbb{R}^{n}$. The notions presented above are naturally extended to the mappings $f: D \rightarrow \overline{\mathbb{R}^{n}}$, where $\overline{\mathbb{R}^{n}}=\mathbb{R}^{n} \cup\{\infty\}$ is a one-point compactification of $\mathbb{R}^{n}$. A Borel function $\rho: \mathbb{R}^{n} \rightarrow[0, \infty]$ is called admissible for a family $\Gamma$ of curves $\gamma$ in $\mathbb{R}^{n}$ if $\int_{\gamma} \rho(x)|d x| \geq 1$ for all curves $\gamma \in \Gamma$. In this case, we write $\rho \in \operatorname{adm} \Gamma$. The modulus of a family of curves $\Gamma$ is defined as follows:

$$
M(\Gamma)=\inf _{\rho \in \operatorname{adm} \Gamma} \int_{D} \rho^{n}(x) d m(x) .
$$

Let $E, F \subset \overline{\mathbb{R}^{n}}$ be arbitrary sets. By $\Gamma(E, F, D)$ we denote the family of all curves $\gamma:[a, b] \rightarrow \overline{\mathbb{R}^{n}}$ connecting $E$ with $F$ in $D$, i.e., $\gamma(a) \in E, \gamma(b) \in F$, and $\gamma(t) \in D$ for $t \in(a, b)$.

In [7, Sec. 13], Gehring defined a $K$-quasiconformal mapping as a homeomorphism changing the modulus of an annular domain by at most a factor of $K$. In view of the facts presented above, we introduce a new class of mappings (see, e.g., [2, Sec. 7.1, Chap. VII]. Let $r_{0}=\operatorname{dist}\left(x_{0}, \partial D\right)$, let $Q: D \rightarrow[0, \infty]$ be a Lebesgue measurable function, and let $S_{i}:=S\left(x_{0}, r_{i}\right)$. We say that $f: D \rightarrow \overline{\mathbb{R}^{n}}$ is a ring $Q$-mapping at a point $x_{0} \in D$ if the inequality

$$
M\left(f\left(\Gamma\left(S_{1}, S_{2}, A\right)\right)\right) \leq \int_{A} Q(x) \cdot \eta^{n}\left(\left|x-x_{0}\right|\right) d m(x)
$$

holds for any ring $A=A\left(r_{1}, r_{2}, x_{0}\right), 0<r_{1}<r_{2}<r_{0}$, and any measurable function $\eta:\left(r_{1}, r_{2}\right) \rightarrow[0, \infty]$ such that

$$
\int_{r_{1}}^{r_{2}} \eta(r) d r \geq 1
$$

We say that $f$ is a ring $Q$-mapping in the domain $D$ if relations (4) and (5) are true for $f$ at every point $x_{0}$ of the domain $D$. The word "ring" in this definition reflects the origin of the family of curves $\Gamma\left(S_{1}, S_{2}, A\right)$ appearing on the left-hand side of inequality (4) and the word " $Q$-mapping" corresponds to a given real-valued function $Q$ on the right-hand side of inequality (4).

The investigation of the ring $Q$-mappings is, in particular, connected with the study of the Sobolev classes and with the analysis of Beltrami-type equations whose solutions satisfy the relations of the form (4) (see, e.g., [2, Sec. 11.5, Chap. XI]). Note that the $K$-quasiconformal mappings satisfy relations of the form (3), (4) for $Q(x) \equiv$ $K \in[1, \infty)$. Conversely, if $f$ is a homeomorphism satisfying the relation of the form (3) for $Q(x) \equiv K \in[1, \infty)$, then $f$ is a $K$-quasiconformal mapping (see [1, Theorem 34.3]).

In the case where an open discrete mapping $f$ satisfies a relation of the form (3) for $Q(x) \equiv K \in[1, \infty)$, the function $Q$ can be removed from the integrand in (3) and, hence, the right-hand side of (3) contains the modulus of $\Gamma$ multiplied by $K$. In this case, $f$ is called a mapping with bounded distortion. In more detail, the definitions and properties of quasiregular mappings (or mappings with bounded distortion) can be found, e.g, in [8, 9]. Note that the main difficulty encountered in proving a property of the mapping $f$ satisfying a relation of the form (3) or (4) for an unbounded function $Q(x)$ is connected with the necessity of tracing the behavior of the right-hand 
side of the corresponding inequality depending on the behavior of the function $Q$. Clearly, these difficulties do not appear for mappings with bounded distortion.

The following important definitions can be found in [9, Sec. 3, Chap. II]: Let $f: D \rightarrow \mathbb{R}^{n}$, let $\beta:[a, b) \rightarrow \mathbb{R}^{n}$ be a curve, and let $x \in f^{-1}(\beta(a))$. A curve $\alpha:[a, c) \rightarrow D$ is called the maximum elevation of the curve $\beta$ under the mapping $f$ with origin at the point $x$ whenever

(i) $\alpha(a)=x$;

(ii) $f \circ \alpha=\left.\beta\right|_{[a, c)}$;

(iii) if $c<c^{\prime} \leq b$, then there is no curve $\alpha^{\prime}:\left[a, c^{\prime}\right) \rightarrow D$ such that $\alpha=\left.\alpha^{\prime}\right|_{[a, c)}$ and $f \circ \alpha^{\prime}=\left.\beta\right|_{\left[a, c^{\prime}\right)}$.

Let $f$ be an open discrete mapping and let $x \in f^{-1}(\beta(a))$. Then the curve $\beta:[a, b) \rightarrow \mathbb{R}^{n}$ has the maximum elevation under the mapping $f$ with origin at the point $x$ (see [9, Corollary 3.3, Chap. II]). A capacitor in $\mathbb{R}^{n}, n \geq 2$, is defined as a couple $E=(A, C)$, where $A$ is an open set from $\mathbb{R}^{n}$ and $C$ is a compact subset of the set $A$. Recall that a mapping $f: D \rightarrow \mathbb{R}^{n}$ is called absolutely continuous on lines (in this case, we write $f \in A C L)$ if all coordinate functions $f=\left(f_{1}, \ldots, f_{n}\right)$ are absolutely continuous in almost all lines parallel to the coordinate axes for any $n$-dimensional parallelepiped $P$ whose edges are parallel to the coordinate axes (such that $\bar{P} \subset D$ ). It is known that if $f \in A C L$, then $f$ has partial derivatives almost everywhere in $D$. The capacity of the capacitor $E$ is defined as follows:

$$
\operatorname{cap} E=\operatorname{cap}(A, C)=\inf _{u \in W_{0}(E)} \int_{A}|\nabla u|^{n} d m(x),
$$

where

$$
W_{0}(E)=W_{0}(A, C)
$$

is a family of nonnegative continuous functions $u: A \rightarrow \mathbb{R}$ with compact support in $A$ such that $u(x) \geq 1$ for $x \in C$ and $u \in A C L$.

For a mapping $f: D \rightarrow \mathbb{R}^{n}$ whose partial derivatives exist almost everywhere in the domain $D$, let $f^{\prime}(x)$ be the Jacobian matrix of the mapping $f$ at a point $x$ and let

$$
\left\|f^{\prime}(x)\right\|=\max _{h \in \mathbb{R}^{n} \backslash\{0\}} \frac{\left|f^{\prime}(x) h\right|}{|h|} .
$$

The outer dilatation of the mapping $f$ at the point $x$ is the quantity

$$
K_{O}(x, f)=\frac{\left\|f^{\prime}(x)\right\|^{n}}{|J(x, f)|}
$$

for $J(x, f) \neq 0, K_{O}(x, f)=1$ for $f^{\prime}(x)=0$, and $K_{O}(x, f)=\infty$ at all other points. The linear dilatation of a mapping $f$ at a point $x$ is defined as follows:

$$
H(x, f)=\sqrt[n]{K_{I}(x, f) K_{O}(x, f)}
$$

It is known (see, e.g., [8, Sec. 3, Chap. I]) that

$$
K_{I}(x, f) \leq K_{O}^{n-1}(x, f), \quad K_{O}(x, f) \leq K_{I}^{n-1}(x, f)
$$


In [10], the $A C L$ property is established for the homeomorphisms in $\mathbb{R}^{n}, n \geq 2$, satisfying a relation of the form (3) for a locally integrable function $Q$. In the same paper, it is also shown that these homeomorphisms belong to the class $W_{\mathrm{loc}}^{1,1}$ and are differentiable almost everywhere. The following estimate of the outer dilatation valid for almost all $x \in D$ was also established in [10]:

$$
K_{O}(x, f) \leq C_{n} \cdot Q^{n-1}(x) .
$$

Moreover, the open discrete mappings $f: D \rightarrow \overline{\mathbb{R}^{n}}$ satisfying an estimate of the form (4) in the domain $D$ for any nonnegative measurable function $\eta$ satisfying the "normalization" condition (5) and a function $Q \in L_{\text {loc }}^{1}(D)$ are differentiable almost everywhere [11, Theorem 3.1] and satisfy estimate (6) [11, Corollary 3.2]. In particular, the arguments presented above imply that the inner $K_{I}(x, f)$ and outer $K_{O}(x, f)$ dilatations for the open discrete ring $Q$-mappings $f$ are correctly defined at least under the condition of local integrability of the function $Q(x)$ on the right-hand side of the determining relation (4) provided that $J(x, f) \neq 0$ almost everywhere (see [11]).

It is known that, for the capacitor $E=(A, C)$,

$$
\operatorname{cap} E \geq \frac{\left(\inf m_{n-1} S\right)^{n}}{[m(A \backslash C)]^{n-1}},
$$

where $m_{n-1} S$ is the $(n-1)$-dimensional Lebesgue measure of the $C^{\infty}$-manifold $S$ obtained as the boundary $S=\partial U$ of a bounded open set $U$ containing the set $C$ and contained, together with its closure $\bar{U}$, in the set $A$. In (7), the infimum is taken over all $S$ of this sort (see [12, Proposition 5]).

Proposition 3. Let $E=(A, C)$ be an arbitrary capacitor in $\mathbb{R}^{n}$ and let $\Gamma_{E}$ be the family of all curves of the form $\gamma:[a, b) \rightarrow A$ with $\gamma(a) \in C$ and $|\gamma| \cap(A \backslash F) \neq \varnothing$ for any compact set $F \subset A$. Then cap $E=M\left(\Gamma_{E}\right)$ (see [9, Proposition 10.2, Chap. II]).

\section{Formulation and Proof of the Main Result. Corollaries}

Theorem 1. Let $f: D \rightarrow \overline{\mathbb{R}^{n}}$ be an open discrete ring $Q$-mapping. Assume that $Q \in L_{\mathrm{loc}}^{1}(D)$ and $J(x, f) \neq 0$ almost everywhere. Then the following relation holds for almost all $x \in D$ :

$$
K_{I}(x, f) \leq c_{n} \cdot Q(x),
$$

where $c_{n}$ is a constant depending only on $n$.

Proof. According to [11, Theorem 3.1], the mapping $f$ is differentiable almost everywhere in the domain $D$. Without loss of generality, we can assume that $\infty \notin D^{\prime}=f(D)$ and $J(x, f)>0$ at all points where the derivative of $f$ exists and is not equal to zero and, in addition, $J(x, f) \neq 0$. At every point $x \in D$ of differentiability of the mapping $f$ such that $J(x, f) \neq 0$, we consider a capacitor $E_{r}=\left(A_{r}, G_{r}\right)$, where $A_{r}=\{y:|x-y|<2 r\}$ and $G_{r}=\{y:|x-y| \leq r\}$. Since $f$ is an open continuous mapping, $f\left(E_{r}\right)$ is also a capacitor in $\mathbb{R}^{n}$. Let $\Gamma_{E_{r}}$ and $\Gamma_{f\left(E_{r}\right)}$ be the families of curves in a sense of Proposition 1 and let $\Gamma_{r}^{*}$ be the family of maximum elevations of $\Gamma_{f\left(E_{r}\right)}$ under the mapping $f$ with origin at $G_{r}$. We now show that $\Gamma_{r}^{*} \subset \Gamma_{E_{r}}$.

Assume the contrary, i.e., that there exists a curve $\beta:[a, b) \rightarrow \mathbb{R}^{n}$ from the family $\Gamma_{f\left(E_{r}\right)}$ for which the corresponding maximum elevation $\alpha:[a, c) \rightarrow A_{r}$ lies in a certain compact set $K$ inside $A_{r}$. Hence, its closure $\bar{\alpha}$ is a compact subset of $A_{r}$. Note that $c \neq b$ because otherwise $\bar{\beta}$ is a compact subset of $f\left(A_{r}\right)$, which contradicts the condition $\beta \in \Gamma_{f\left(E_{r}\right)}$. We now consider the set

$$
G=\left\{x \in \mathbb{R}^{n}: x=\lim _{k \rightarrow \infty} \alpha\left(t_{k}\right)\right\}, \quad t_{k} \in[a, c), \quad \lim _{k \rightarrow \infty} t_{k}=c .
$$


Note that, in this case, we can pass to subsequences and, thus, restrict ourselves to the monotone sequences $t_{k}$. For $x \in G$, in view of the continuity of the mapping $f$, we get $f\left(\alpha\left(t_{k}\right)\right) \rightarrow f(x)$ as $k \rightarrow \infty$, where $t_{k} \in[a, c)$ and $t_{k} \rightarrow c$ as $k \rightarrow \infty$. However,

$$
f\left(\alpha\left(t_{k}\right)\right)=\beta\left(t_{k}\right) \rightarrow \beta(c) \quad \text { as } \quad k \rightarrow \infty .
$$

Hence, we conclude that $f$ is constant on $G$ in $A_{r}$. On the other hand, by the Cantor condition, for a compact set $\bar{\alpha}$ (see $[13$, Sec. 3.6, Chap. I]), we have

$$
G=\bigcap_{k=1}^{\infty} \overline{\alpha\left(\left[t_{k}, c\right)\right)}=\limsup _{k \rightarrow \infty} \alpha\left(\left[t_{k}, c\right)\right)=\liminf _{k \rightarrow \infty} \alpha\left(\left[t_{k}, c\right)\right) \neq \varnothing
$$

by virtue of monotonicity relative to the sequence of connected sets $\alpha\left(\left[t_{k}, c\right)\right)$ and, therefore, $G$ is connected according to [13, Sec. 9.12, Chap. I]. Thus, in view of the discreteness of the mapping $f$, the set $G$ cannot contain more than one point, the curve $\alpha:[a, c) \rightarrow A_{r}$ is extended to the closed curve $\alpha:[a, c] \rightarrow K \subset A_{r}$, and $f(\alpha(c))=\beta(c)$. Once again, according to [9, Corollary 3.3, Chap. II], we can construct the maximum elevation $\alpha^{\prime}$ of the curve $\left.\beta\right|_{[c, b)}$ with origin at the point $\alpha(c)$. Combining the elevations $\alpha$ and $\alpha^{\prime}$, we get a new elevation $\alpha^{\prime \prime}$ of the curve $\beta$ defined on $\left[a, c^{\prime}\right), c^{\prime} \in(c, b)$, which contradicts the maximality of the elevation $\alpha$. Thus, $\Gamma^{*} \subset \Gamma_{E_{r}}$. Note that $\Gamma_{f\left(E_{r}\right)}>f\left(\Gamma_{r}^{*}\right)$, and, hence, according to Proposition 3,

$$
\operatorname{cap} f\left(E_{r}\right)=M\left(\Gamma_{f\left(E_{r}\right)}\right) \leq M\left(f\left(\Gamma_{r}^{*}\right)\right) \leq M\left(f\left(\Gamma_{E_{r}}\right)\right)
$$

Since $f$ is a ring $Q$-mapping in the domain $D$, it follows from (8) that

$$
\operatorname{cap} f\left(E_{r}\right) \leq \int_{r<|x-y|<2 r} Q(y) \eta^{n}(|x-y|) d m(y)
$$

for any nonnegative measurable function $\eta:(r, 2 r) \rightarrow[0, \infty]$ such that

$$
\int_{r}^{2 r} \eta(t) d t \geq 1
$$

In particular, we consider the following one-parameter family of real-valued functions

$$
\eta_{r}(t)= \begin{cases}\frac{1}{r} & \text { for } \quad t \in(r, 2 r) \\ 0 & \text { for } \quad t \in \mathbb{R} \backslash(r, 2 r)\end{cases}
$$

Then

$$
\text { cap } f\left(E_{r}\right) \leq \frac{2^{n} \Omega_{n}}{m\left(A_{r}\right)} \int_{A_{r}} Q(y) d m(y)
$$


On the other hand, according to inequality (7), we have

$$
\operatorname{cap} f\left(E_{r}\right) \geq \frac{\left(\inf m_{n-1} S\right)^{n}}{\left[m\left(f\left(A_{r}\right) \backslash f\left(G_{r}\right)\right)\right]^{n-1}},
$$

where the infimum is taken over all possible $C^{\infty}$-manifolds $S$ that form the boundary $S=\partial U$ of a bounded open set $U$ containing $f\left(G_{r}\right)$ and contained, together with its closure $\bar{U}$, in $f\left(A_{r}\right)$. Combining (9) and (10), we get

$$
\left(\inf m_{n-1} S\right)^{n} \leq \frac{2^{n} \Omega_{n}\left[m\left(f\left(A_{r}\right) \backslash f\left(G_{r}\right)\right)\right]^{n-1}}{m\left(A_{r}\right)} \int_{A_{r}} Q(y) d m(y) .
$$

As $r \rightarrow 0$, the set $f\left(G_{r}\right)$ turns into an ellipsoid $f^{\prime}\left(G_{r}\right)$ (to within $o(r)$ ), i.e., the image of the ball $G_{r}$ under the linear mapping $f^{\prime}$. If this ellipsoid has the semiaxes $0<a_{1} r \leq \ldots \leq a_{n} r$, then

$$
m\left(f^{\prime}\left(G_{r}\right)\right)=\Omega_{n} a_{1} \ldots a_{n} r^{n}=\Omega_{n} J(x, f) r^{n} .
$$

We place this ellipsoid so that its center coincides with the the origin of coordinates and its principal directions coincide with the coordinate axes $e_{1}, \ldots, e_{n}$. Then its surface area admits the following lower bound:

$$
m_{n-1}\left(\partial f^{\prime}\left(G_{r}\right)\right) \geq 2 m_{n-1}\left(\operatorname{Pr}_{1}\left(f^{\prime}\left(G_{r}\right)\right)\right)=2 \Omega_{n-1} \cdot a_{2} \ldots a_{n} r^{n-1}=2 \Omega_{n-1} \frac{J(x, f)}{l\left(f^{\prime}(x)\right)} r^{n-1},
$$

where $\operatorname{Pr}_{1}(\cdot)$ denotes the projection onto the hyperplane perpendicular to the vector $e_{1}$. Consequently, according to (11) and (12), we get

$$
\begin{aligned}
{\left[2 \Omega_{n-1} \frac{J(x, f)}{l\left(f^{\prime}(x)\right)} r^{n-1}-o\left(r^{n-1}\right)\right]^{n} } & \leq\left[m_{n-1} \partial f^{\prime}\left(G_{r}\right)-o\left(r^{n-1}\right)\right]^{n} \\
& \leq \frac{2^{n} \Omega_{n}\left[m\left(f\left(A_{r}\right) \backslash f\left(G_{r}\right)\right)\right]^{n-1}}{m\left(A_{r}\right)} \int_{A_{r}} Q(y) d m(y) .
\end{aligned}
$$

Dividing inequality (13) by $r^{n(n-1)}$, passing to the limit as $r$ approaches 0 , and applying the Lebesgue theorem on differentiability of indefinite integrals (see [14]), we find

$$
\left[\frac{J(x, f)}{l\left(f^{\prime}(x)\right)}\right]^{n} \leq[J(x, f)]^{n-1} c_{n} \cdot Q(x)
$$

for almost all $x \in D$. Note that, by the assumption of Theorem 1 , the condition $J(x, f) \neq 0$ is true almost everywhere. Thus, we obtain

$$
K_{I}(x, f)=\frac{J(x, f)}{\left(l\left(f^{\prime}(x)\right)\right)^{n}} \leq c_{n} \cdot Q(x)
$$

for almost all $x \in D$. 
Corollary 1. Let $f: D \rightarrow \overline{\mathbb{R}^{n}}$ be an open discrete ring $Q$-mapping in the domain $D$. Assume that $Q \in$ $L_{\mathrm{loc}}^{1}(D)$ and $J(x, f) \neq 0$ almost everywhere. Then

$$
H(x, f) \leq c_{n} \cdot Q(x)
$$

almost everywhere, where the constant $c_{n}$ depends only on $n$.

Corollary 2. Let $f: D \rightarrow \overline{\mathbb{R}^{n}}$ be an open discrete ring $Q$-mapping in the domain $D$. Assume that $Q \in$ $L_{\mathrm{loc}}^{1}(D)$ and $J(x, f) \neq 0$ almost everywhere. Then $H(x, f) \in L_{\mathrm{loc}}^{1}(D)$ and $K_{I}(x, f) \in L_{\mathrm{loc}}^{1}(D)$.

Corollary 3. If, under the conditions of Theorem 1 and Corollaries 1 and 2, the mapping $f$ additionally satisfies a stronger estimate of the form (3) for arbitrary curves $\gamma$ from the family $\Gamma$ and $\rho \in \operatorname{adm} \Gamma$, then the corresponding assertions remain true almost everywhere even without the additional condition $J(x, f) \neq 0$. The last condition automatically follows from relation (3) for $Q \in L_{\mathrm{loc}}^{1}$ (see, e.g., [11, Corollary 3.5]).

\section{REFERENCES}

1. J. Väisälä, Lectures on n-Dimensional Quasiconformal Mappings, Springer, Berlin (1971).

2. O. Martio, V. Ryazanov, U. Srebro, and E. Yakubov, Moduli in Modern Mapping Theory, Springer, New York (2009).

3. O. Lehto and K. Virtanen, Quasiconformal Mappings in the Plane, Springer, New York (1973).

4. Yu. F. Strugov, "Compactness of the families of mappings quasiconformal in the mean," Dokl. Acad. Nauk SSSR, 243, No. 4, 859-861 (1978).

5. C. J. Bishop, V. Ya. Gutlyanskii, O. Martio, and M. Vuorinen, “On conformal dilatation in space,” Int. J. Math. Math. Sci., 22, 1397-1420 (2003).

6. V. M. Miklyukov, Conformal Mappings of Irregular Surfaces and Their Applications [in Russian], Volgograd University, Volgograd (2005).

7. F. W. Gehring, "Rings and quasiconformal mappings in space," Trans. Amer. Math. Soc., 103, 353-393 (1962).

8. Yu. G. Reshetnyak, Space Mappings with Bounded Distortion [in Russian], Nauka, Novosibirsk (1982).

9. S. Rickman, "Quasiregular mappings," Results Math. Relat. Areas, 26, No. 3 (1993).

10. R. Salimov, "Absolute continuity on lines and the differentiability of one generalization of quasiconformal mappings," Izv. Ross. Acad. Nauk, Ser. Mat., 72, No. 5, 141-148 (2008).

11. R. R. Salimov and E. A. Sevost'yanov, "Theory of ring $Q$-mappings in geometric function theory," Mat. Sb., 201, No. 6, 131-158 (2010).

12. V. I. Kruglikov, "Capacities of capacitors and space mappings quasiconformal in the mean,” Mat. Sb. 130, No. 2, 185-206 (1986).

13. G. T. Whyburn, Analytic Topology, American Mathematical Society, Providence, RI (1942).

14. S. Saks, Theory of the Integral, Dover, New York (1937). 